\title{
Smart Parking System with IoT involving Disciplined parking mechanism
}

\author{
Annapurna H S ${ }^{1}$, Spoorthi $\mathrm{N}^{2}$, Samved Purohit ${ }^{3}$, Sanjeev Kumar ${ }^{4}$, Divya $\mathrm{M} \mathrm{N}^{5}$ \\ Electronics and Communication Engineering, \\ REVA University Bangalore, India \\ Email : annapurnaathri@gmail.com ${ }^{1}$, spoorthinagaraj8@gmail.com ${ }^{2}$, purohitsamved@gmail.com ${ }^{3}$, sanjeevkumar9511@gmail.com ${ }^{4}$, \\ divya.mn@ reva.edu.in ${ }^{5}$
}

\begin{abstract}
Most metropolitan cities have seen a rapid increase in automobile population in the last decade. Due to this, finding a place to park a vehicle has become rather hectic and time consuming. This paper, Smart parking system with IoT involving disciplined parking mechanism, aims to reduce the time taken to find a parking spot for your automobile. This will also reduce the stress on the driver and will help in conserving fuel. It uses a web application to get real time sensor data on any screen. This reduces the time taken to park the vehicle and the stress on driver while also conserving fuel. A way to help drivers to park their automobiles in a disciplined manner is also inculcated in this paper.
\end{abstract}

Key Words : IoT,

\section{Introduction}

In huge metropolitan cities of India, traffic congestion is a huge problem and finding a parking spot for your automobile has become a rather hectic and time consuming task. The proposed work aims to reduce the time taken to find a spot to park an automobile and also conserve fuel. As a result, this also leads to reduced greenhouse emissions and helps to fight global warming. The proposed Smart parking system with IoT uses Internet of things technology (IoT) to deliver data from the sensors to the cloud. Internet of Things technology is a way to connect, coordinate and access data from devices and objects from remote environment through the internet. Internet of Things (IoT) technology makes any system wireless and any user with an internet connection can access it remotely on their respective device. IoT is one of the most popular technologies during the recent times. Use of IoT enables devices to send data, receive data and in certain occasions, both. This is what makes the smart parking system with IoT "smart".

The devices that are connected to the internet can be sensors. These devices can collect and send information, receive information and act accordingly or can do both simultaneously.

The smart parking system with IoT makes use of a web application to broadcast this data to its users through a Graphical User Interface (GUI). During recent times, web applications have been designed and implemented for innumerable purposes. These web application reduces human effort. One can access real time information on any device with an internet connection from any part of the planet.

Thus the proposed paper consisting of sensors, IoT, Web applications, integrates its uses and provides a smooth experience to a person at the user end.

\section{LITERATURE SURVEY}

The use of Internet of Things technology(IoT) has increased in various application domains like

smart city, increase the progress of quality in environment etc. This has resulted in the generation of enormous volume of data. This has risen privacy issues which has been tackled efficiently in the mentioned paper [1].

In one of the papers, IoT technology (Internet Of Things) has been used in order to provide real time information. The data collected can be service based or a feedback technique to give the user satisfactory experience in various fields of services (e.g., parking, food, healthcare, transportation, and entertainment) [2].

Smart City Application: Android Based Smart Parking System uses wireless sensor network to provide information from different region. Sensors communicate with each other and receive data from surrounding.The information obtain from surrounding is send into network environment [3].

Smart car parking system embedded with Infrared Radiation (IR) sensor and Internet of Things technology(IoT). which allow the user to find the proximate parking slot, and gives the number of free places accessible in the nearest parking zone[4].

Verification of Smart Guiding System to Search for Parking Space via DSRC Communication based on Infrared Radiation (IR) sensor. And by using dedicated short range communication (DSRC) is used to show the exact location of spot on smart phone or tablet pc where user can park their automobile [5]

advanced CAR Parking System uses a card system. If a person wants to park in their parking place they need to 
make card which is used as ID proof for that person and if they do not have a card they are not to park their vehicle. Arduino controller is used to display floor that has a free space in the parking place[6]. Centralized control parking system design an SPS using geeknesia application program interface(API) as Internet of Things (IoT) for embedded system. The system is inserted with integrated internet based monitoring on smart phone[7].

Real Time Car Parking System installed with mobile cloud computing (MCC) and vehicular networking (VN)

\section{Methodology}

The idea of the smart parking system with IoT involving disciplined parking mechanism emerged due to the insufficiency of parking spaces in our city. Hence, wee conducted a survey so that people could address their problems and we could find out what problems other automobile drivers were facing.

Given below is a graph of the survey that was conducted.

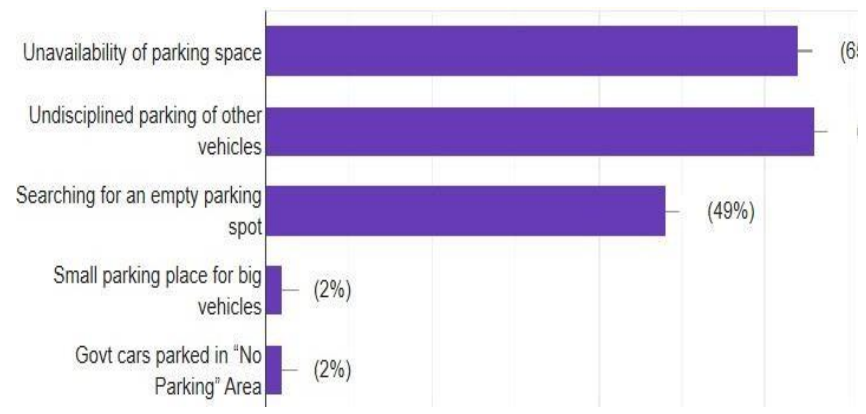

Fig. 1: Survey conducted.

It has been noticed that almost $50 \%$ of the people we surveyed found that searching for empty parking spot has become a very hectic and time consuming task. It was also noticed that $67.3 \%$ of the people also felt that undisciplined parking of other vehicles was causing an inconvenience.

Therefore the proposed work aims to reduce these problems. The general methodology of the smart parking system with IoT involving disciplined parking mechanism is as follows.

The prototype of the system consists of four ultrasonic sensors that help in detection of the automobile at a particular parking spot. This sensor data is sent to a microcontroller, the Node MCU. The sensor state will be logic 1 if there is a automobile at a particular stretch from the threshold and it will be logic 0 if there is no automobile.

This data is driven over to the cloud and made accessible to the users via a web application. The web application consists of a login page for the

Vol. 3 (12), June 2020, www.ijirase.com users and the users can select a spot of their choice and book the required spot.

The smart parking system with IoT also has a disciplined parking system that ensures the disciplined parking of the vehicles as this is one of the main reasons of inconvenience to drivers as proven by the survey that we had conducted. There is also an integrated billing system that the users have to use to pay for the spot in accordance to time for which the parking spot had been occupied.

\section{A. Flow chart of the web application}

The flow chart of the working of the web application has been shown.

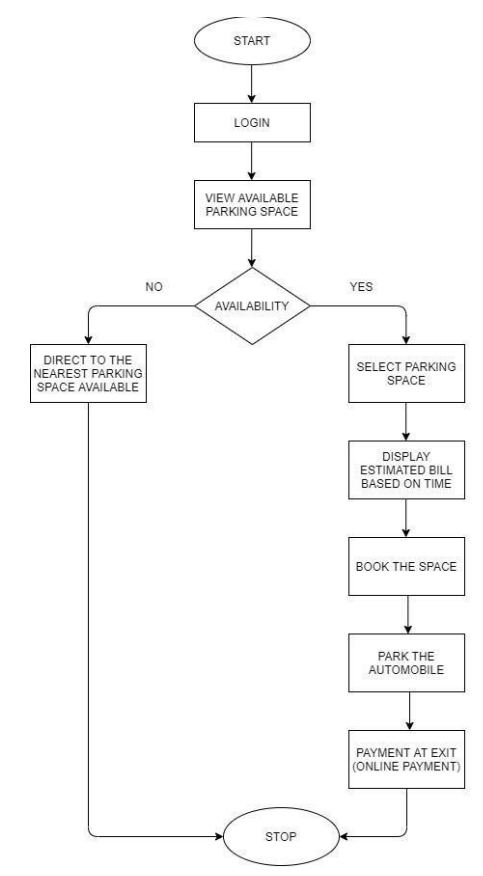

Fig. 2: Flow chart of the web application

The complete understanding of the flowchart can be understood by the following steps.

Step 1: The credentials of the user is entered for booking purposes. The credentials include Name of the user and the phone number of the user.

Step 2: This is one of the most crucial steps that takes place in the back end of the web application. If an empty spot ceases to exist, an automatic message will be sent to the user for the nearest parking lot with an empty parking spot.

Step 3: If a spot for the users' automobile is available, a booking page appears where the user can select a spot as per their convenience and book the spot for their vehicle. Step 4: The timings for which the user wants to park their car will be recorded and an estimated bill will be generated which can be paid while exiting the parking premises.

Step 5: After the automobile is parked and the user wants to leave, an online payment portal is opened where the user can pay for the duration for which they have 
occupied the spot using net banking or credit/debit cards or any other UPI payment methods.

The disciplined parking of vehicles can be achieved by the use of blinking or color changing LED's. In the proposed smart parking system with IoT involving disciplined parking mechanism, we have made use of a color changing LED system. The color of the LED will change from Red to Green when the vehicle is properly parked. If the driver leaves the automobile parked incorrectly an SMS message will be sent to the driver so that the driver will come and park their automobile in a correct way.

Also to prevent vehicles from hitting the sensors, if the vehicle come too close to the sensor (as per decided threshold), an LED will start blinking to indicate to the driver that their automobile might damage the sensor as they are too close to it.

\section{B. Hardware Used}

- $\quad$ Node MCU:

The Node MCU is an open source, low cost firmware. On board the Node MCU, there is a ESP $8266 \mathrm{Wi}-\mathrm{Fi}$ module. The ESP 8266 employs a System on Chip (SoC) technology and is very popular among Internet of Things (IoT) and embedded system applications. The node MCU supports serial communication protocols such as UART, I2C, SPI etc. The node MCU consists of 10 GPIO pins, 2 reserved pins and 3 ground (GND) pin

\section{- HC-SR04 Ultrasonic Sensor:}

The ultrasonic sensors works on a principle of reflection of sound waves. The HC-SR04 has 4 pins- Vcc, Trig, Echo and GND. The ultrasonic sensor used has a minimum range of $2 \mathrm{~cm}$ and a maximum range of $400 \mathrm{~cm}$. The HC-SR04 has a working voltage of 5 volts, a working current of $15 \mathrm{~mA}$ and a working frequency of $40 \mathrm{~Hz}$.

\section{Software Used}

- Arduino IDE:

Arduino IDE is a software used to establish connection between the microcontroller and the sensor. IoT connection is also established through coding on Arduino IDE platform. Installing the necessary libraries for coding the system, the program is written for the devices connected. The code is dumped on to the microcontroller and connection is established. The Arduino IDE uses the languages $\mathrm{C}$ and $\mathrm{C}++$ but consists of a set of rules for structuring of the program. It can be run on Windows, Linux and Mac OS X.

\section{- Sublime Text Editor:}

Sublime Text Editor is a shareware cross- platform source code editor. It supports many programming languages and mark up languages. It can be run on Mac, Windows and Linux. In this project, we have used Sublime Text Editor to build the front end of the web application using HTML and Cascading Style Sheets(CSS).

Bootstrap is an open source CSS framework which has inbuilt styles to design the website. While HTML builds the structure of the web page, CSS gives the web page styles making the web pages more attractive with neat layout and effects.

JavaScript is used for the back end of the web page. This makes the web page responsive and dynamic. JavaScript supports objectoriented programming.

Sublime Text allows codes of different languages and script to run at a time and links one file to another to provide a fully furnished Web page for user interaction.

\section{- Thingspeak cloud:}

The sensor data cannot be directly sent to the web page. In this scenario cloud place an important role. The sensor information is sent to the Node MCU from which it is sent to the cloud through internet using the wi-fi module. From the cloud the sensor status can be accessed and brought down to the web page. The cloud platform we have used in our project is ThingSpeak. This open IoT (Internet of Things) platform, helps us collect data from sensors and visualize the information through graphs.

\section{Equations}

The formula to calculate the distance using the HCSR04 ultrasonic sensor is as shown below.

We are assuming the speed of sound in air at room temperature to be $340 \mathrm{~m} / \mathrm{s}$ or $0.034 \mathrm{~cm} / \mu \mathrm{s}$.

Since we know that,

Distance, $\mathbf{s}=\mathbf{v}^{*} \mathbf{t}$

Where $\mathrm{v}$ is the velocity and $\mathrm{t}$ is the time.

The ultrasonic sensor uses this concept to accurately measure the distance between the object and itself. It calculates the time taken for the ultrasonic sound wave to strike the object and bounce back and accordingly calculates the distance between the sensor and the object.

The formula used to calculate the distance is given as Distance, $\mathbf{d}=($ time taken $* \mathbf{0 . 0 3 4}) / 2$....(2)

It is to be observed that the division by 2 is done because the ultrasonic wave travels twice the distance between the sensor and the object. 


\section{RESUlTS AND DisCUSSIONS}

After the complete rigging up of the circuitry which includes the Node MCU, the ultrasonic sensors, the LED's for disciplined parking purposes, the data can be pushed over to the cloud and can be accessed onto the web application. A few screenshots of the web application and data from the thing speak cloud have been shown below. This is what appears to the user so the he/she can book a parking spot according to their convenience.

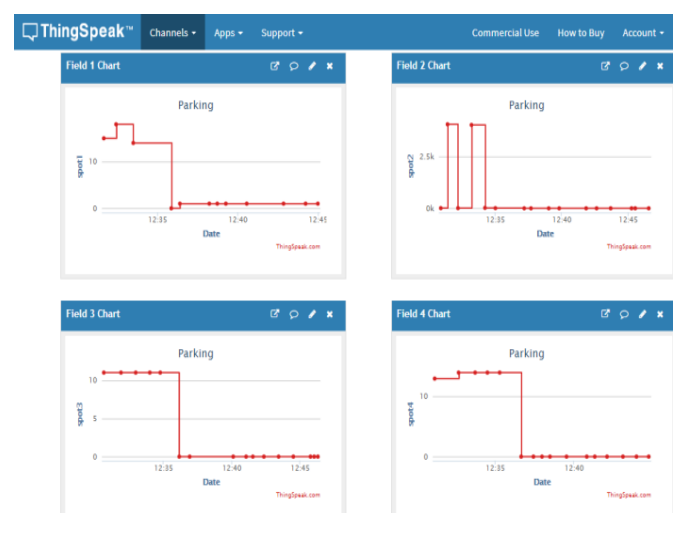

Fig. 3 Thingspeak Cloud

The data uploaded to thingspeak cloud is in the forom of a step signal. The simulated signals are as shown above. The higher peaks indicate that the spots have been occupied and the lower peaks indicate that the spot is empty. Below are some of the screenshots of the web application that we have developed for the advanced parking spot assistance system.

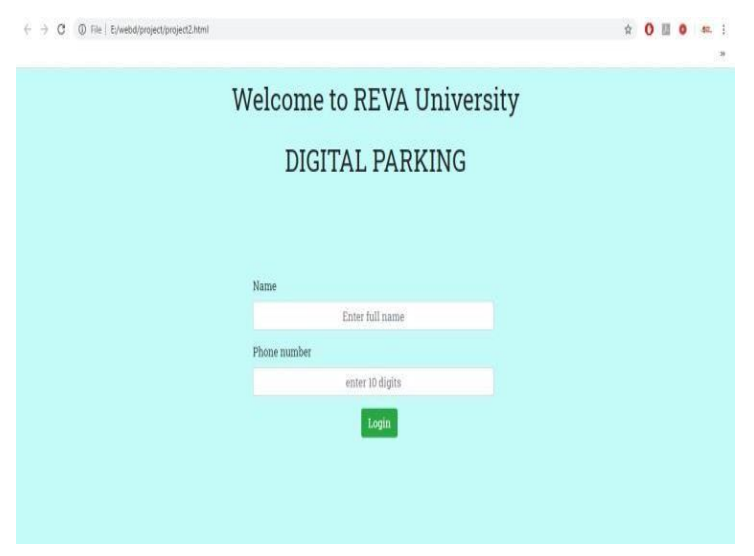

Fig. 4 Login page of web application
The login page of the web application takes the redentials of the user. These credentials include the name of the user and also their phone number.

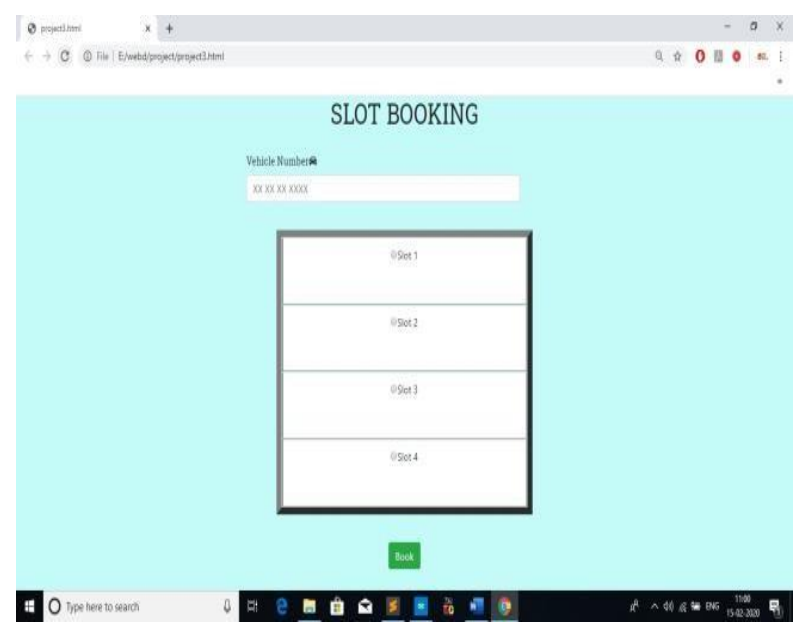

Fig. 5 Slot booking page of web application

This is the slot booking page of the web application. Here the user has to enter in their vehicle number and can select the slot of their choice. In the prototype of the smart parking system with IoT, we have made use of 4 sensors. More number of slots can be added later. Thus in conclusion, the parking spot assistance system can help users book their parking slots and helps them to save time. The web application makes it easier for anyone with a internet connection to access it and helps reduce stress on drivers to find a parking spot.

\section{REFERENCES}

[1] A Study of Enhancing Privacy for Intelligent Transportation Systems: k-Correlation Privacy Model Against Moving Preference Attacks for Location Trajectory Data. PEIPEI SUI, XIANXIAN LI, AND YAN BAI. Received September 15, 2017, accepted October 9, 2017, date of publication October 30, 2017, date of current version November 28, 2017.

[2] Trust Management of Smart Service Communities. PEIPEI SUI , XIANXIAN LI, AND YAN BAI. Received January 21, 2019, accepted February 12, 2019, date of publication February 22, 2019, date of current version March 12, 2019. 
[3] Kilic T and Tuncer T. (2017). Smart city application: Android based smart parking system. 2017 International Artificial Intelligence and Data Processing Symposium (IDAP)

[4] Meenaloshini, M., Ilakkiya, J., Sharmila, P., Sheffi Malar, J. and Nithyasri, S. (2019). Smart Car Parking System in Smart Cities using IR. 2019 3rd International Conference on Computing and Communications Technologies (ICCCT)

[5] Hsu, C. W., Min Huai Shih, Hou Yu Huang, Yu Chi Shiue, \& Shih Chieh Huang. (2012). Verification of smart guiding system to search for parking space via DSRC communication.

[6] Chaudhary, H., Bansal, P., \& Valarmathi, B. (2017). Advanced CAR parking system using Arduino. 2017 4th International Conference on Advanced Computing and Communication Systems (ICACCS).

[7] Mutiara, G. A., Hapsari, G. I., \& Pujud, A. (2018). Centralized Control Parking System using API Geeknesia. 2018 International Conference on Control, Electronics, Renewable Energy and Communications (ICCEREC).

[8] Anderson, E. C., Okafor, K. C., Nkwachukwu, O., \& Dike, D. O. (2017). Real time car parking system: A novel taxonomy for integrated vehicular computing. 2017 International Conference on Computing Networking and Informatics (ICCNI). 